\title{
THE UNIQUENESS PROBLEM FOR MEROMORPHIC FUNCTIONS IN THE UNIT DISC SHARING VALUES AND A SET IN AN ANGULAR DOMAIN
}

\author{
HONG-YAN XU ${ }^{\dagger}$, CAI-FENG YI and TING-BIN CAO*
}

\begin{abstract}
We investigate the uniqueness problem for meromorphic functions in the unit disc sharing four distinct values and one set in an angular domain, and obtain some relations between the Borel points and shared values of meromorphic functions in an angular domain. These results improve the theorem of Mao-Liu [10].
\end{abstract}

\section{Introduction and main results}

We use $C$ to denote the open complex plane, $\widehat{C}(=C \bigcup\{\infty\})$ to denote the extended complex plane, $\mathrm{D}=\{z:|z|<1\}$ to denote the unit disc, and $X$ $(\subseteq \mathrm{C})$ to denote an angular domain. Let $g$ be a transcendental meromorphic function defined in the whole complex plane $\mathrm{C}$ and not rational. It is assumed that the readers are familiar with the notations of the Nevanlinna theory such as $T(r, f), m(r, f), N(r, f)$ and so on, that can be found, for instance, in [5], [19].

Let $S$ be a set of distinct elements in $\widehat{\mathrm{C}}$. Define

$$
\begin{aligned}
& E_{\mathrm{D}}(S, f)=\bigcup_{a \in S}\left\{z \in \mathrm{D} \mid f_{a}(z)=0, \text { counting multiplicities }\right\}, \\
& \bar{E}_{\mathrm{D}}(S, f)=\bigcup_{a \in S}\left\{z \in \mathrm{D} \mid f_{a}(z)=0, \text { ignoring multiplicities }\right\},
\end{aligned}
$$

where $f_{a}(z)=f(z)-a$ if $a \in \mathrm{C}$ and $f_{\infty}(z)=1 / f(z)$.

Let $f$ and $g$ be two non-constant meromorphic functions in C. If $E_{\mathrm{D}}(S, f)=$ $E_{\mathrm{D}}(S, g)$, we say $f$ and $g$ share the set $S C M$ (counting multiplicities) in

\footnotetext{
* This work was supported by the NNSF of China (No. 10871108), the Natural Science Foundation of Jiang-Xi Province in China (Grant No. 2010GQS0119) and the Youth Foundation of Education Department of Jiangxi (No. GJJ10050 and No. GJJ10223) of China.

${ }^{\dagger}$ Hong-Yan $\mathrm{Xu}$ is the corresponding author.

Received 1 March 2010.
} 
D. If $\bar{E}_{\mathrm{D}}(S, f)=\bar{E}_{\mathrm{D}}(S, g)$, we say $f$ and $g$ share the set $S I M$ (ignoring multiplicities) in D. In particular, when $S=\{a\}$, where $a \in \widehat{\mathrm{C}}$, we say $f$ and $g$ share the value $a C M$ in D if $E_{\mathrm{D}}(S, f)=E_{\mathrm{D}}(S, g)$, and we say $f$ and $g$ share the value $a I M$ in $\mathrm{D}$ if $\bar{E}_{\mathrm{D}}(S, f)=\bar{E}_{\mathrm{D}}(S, g)$. Similarly, we use the notations $E_{X}(S, f), \bar{E}_{X}(S, f)$, and if $X=\mathrm{C}$, write simply $E(S, f), \bar{E}(S, f)$ and so on (see [18]).

R. Nevanlinna [11] proved the following well known theorems.

THEOREM 1.1 ([11]). If $f$ and $g$ are two non-constant meromorphic functions that share five distinct values $a_{1}, a_{2}, a_{3}, a_{4}, a_{5} I M$ in $X=\mathrm{C}$, then $f(z) \equiv g(z)$.

THEOREM 1.2 ([11]). If $f$ and $g$ are two distinct non-constant meromorphic functions that share four distinct values $a_{1}, a_{2}, a_{3}, a_{4} C M$ in $X=\mathrm{C}$, then $f$ is a Möbius transformation of $g$, two of the shared values, say $a_{1}$ and $a_{2}$ are Picard values, and the cross ratio $\left(a_{1}, a_{2}, a_{3}, a_{4}\right)=-1$.

In 1993, M. Reinders [12] investigated the uniqueness problem of nonconstant meromorphic functions sharing four distinct values $I M$ and obtained the following result.

THEOREM 1.3 ([12]). Let $f$ and $g$ be distinct non-constant meromorphic functions sharing four distinct values $a_{j}(j=1,2,3,4)$ IM. If there exists $a, b \in \widehat{\mathrm{C}} \backslash\left\{a_{1}, a_{2}, a_{3}, a_{4}\right\}$ such that $f(z)=a \Longrightarrow g(z)=b$, then either $f(z)$ and $g(z)$ are the functions $f=L \circ \hat{f} \circ h$ and $g=L \circ \hat{g} \circ h$ or $f=T \circ g$, where $L, T$ are Möbius transformation, $h$ is a non-constant entire function and

$$
\hat{f}=\frac{e^{z}+1}{\left(e^{z}-1\right)^{2}}, \quad \hat{g}=\frac{\left(e^{z}+1\right)^{2}}{8\left(e^{z}-1\right)} .
$$

After their work, the uniqueness of meromorphic functions with shared values in the whole complex plane attracted many investigations (see [18]). It is an interesting topic to investigate the uniqueness with shared values in the remaining part of the complex plane removing an unbounded closed set, see [2], [3], [8]-[10], [15]-[17], [21], [22]. In [21], Zheng studied the uniqueness problem under the condition that five values are shared in some angular domain in C. Zheng J. H. [22], Cao T. B. and Yi H. X. [3], Xu J. F. and Yi H. X. [17] continued to investigate the uniqueness of meromorphic functions sharing five values and four values in an angular domain, Lin W. C., Mori S. and Tohge K. [8] and Lin W. C., Mori S. and Yi H. X. [9] investigated the uniqueness of meromorphic and entire functions sharing sets in an angular domain. They obtained some important results. 
In 2009, Cao T. B. and Yi H. X. [3] investigated the uniqueness problem of two transcendental meromorphic functions sharing five distinct values in an angular domain and obtained the following theorem:

THeOREM 1.4 ([3, Theorem 1.3]). Let $f$ and $g$ be two transcendental meromorphic functions. Given an angular domain $X=\{z: \alpha<\arg z<\beta\}$ with $0<\beta-\alpha \leq 2 \pi$, we assume that $f$ and $g$ share five distinct values $a_{j} \in \widehat{C}$ $(j=1,2,3,4,5)$ IM in $X$. Then $f(z) \equiv g(z)$, provided that

$$
\lim _{r \rightarrow \infty} \frac{S_{\alpha, \beta}(r, f)}{\log (r T(r, f))}=\infty \quad(r \notin E),
$$

where $S_{\alpha, \beta}(r, f)$ denotes the angular characteristic function of the meromorphic function $f$.

Remark 1.1. We refer to Theorem 1.4 as the $5 I M$ theorem in an angular domain.

In 2009, Zhang [20] proved the following theorems by setting up the relationship between the Nevanlinna angular characteristic function and the Ahlfors angular characteristic function, and applying it to study the uniqueness problems of meromorphic functions in an angular domain.

THEOREM 1.5 ([20]). Let $f, g$ be two meromorphic functions of finite order in $\mathrm{C}$, let $a_{j} \in \widehat{\mathrm{C}}(j=1,2, \ldots, 5)$ be five distinct values, and let $\Delta_{\delta}=\{z$ : $\left.\left|\arg z-\theta_{0}\right| \leq \delta\right\}(0<\delta<\pi)$ be an angular domain satisfying

$$
\varlimsup_{\varepsilon \rightarrow 0^{+}} \varlimsup_{r \rightarrow+\infty} \frac{\log T\left(r, \Delta_{\delta-\varepsilon}, f\right)}{\log r}>\omega,
$$

where $\omega=\frac{\pi}{2 \delta}$ and $T\left(r, \Delta_{\delta-\varepsilon}, f\right)$ denotes the Ahlfors characteristic function of $f$ in $\Delta_{\delta-\varepsilon}$. If $f$ and $g$ share $a_{j}(j=1,2, \ldots, 5)$ IM in $\Delta_{\delta}$, then $f \equiv g$.

In [4], Fang studied the uniqueness problem of admissible meromorphic functions in the unit disc D sharing two sets and three sets. It is also an interesting topic to investigate the uniqueness of meromorphic functions in the unit disc D (see [4], [10], [13]). To state some uniqueness theorems for meromorphic functions in the unit disc $\mathrm{D}$, we need the following basic notations and definitions of meromorphic functions in $\mathrm{D}$.

Definition 1.1 ([6]). A meromorphic function $f$ in $\mathrm{D}$ is said to be admissible if and only if

$$
\limsup _{r \rightarrow 1^{-}} \frac{T(r, f)}{\log \frac{1}{1-r}}=\infty
$$


and non-admissible if and only if

$$
\limsup _{r \rightarrow 1^{-}} \frac{T(r, f)}{\log \frac{1}{1-r}}<\infty .
$$

Let $f(z)$ be a meromorphic function in the unit disc $\mathrm{D}$ and let $\Delta\left(\theta_{0}, \delta\right)$ denote the domain $\{z:|z|<1\} \bigcap\left\{z:\left|\arg z-\theta_{0}\right|<\delta\right\}$, where $0 \leq \theta_{0} \leq 2 \pi$, $0<\delta<\pi$. We use $n\left(r, \Delta\left(\theta_{0}, \delta\right), f(z)=a\right)$ to denote the number of zeros of $f(z)-a$ in $\Delta\left(\theta_{0}, \delta\right) \bigcap\{z:|z|<r\}$ counting multiplicities.

In 2005, F. Titzhoff [13] investigated the uniqueness problem for admissible functions in the unit disc $D$ and obtained the five-values theorem in the unit disc $\mathrm{D}$ as follows.

THEOREM 1.6 ([13]). If two admissible functions $f, g$ share five distinct values, then $f \equiv g$.

In 2009, Z.-Q. Mao and H.-F. Liu [10] gave a different method to investigate the uniqueness problem of meromorphic functions in the unit disc and obtained the following results.

THEOREM 1.7 ([10]). Let $f, g$ be two meromorphic functions in $\mathrm{D}, a_{j} \in \widehat{\mathrm{C}}$ $(j=1,2, \ldots, 5)$ be five distinct values, and $\Delta\left(\theta_{0}, \delta\right)(0<\delta<\pi)$ be an angular domain such that for some $a \in \widehat{\mathrm{C}}$,

$$
\limsup _{r \rightarrow 1^{-}} \frac{\log n\left(r, \Delta\left(\theta_{0}, \delta / 2\right), f(z)=a\right)}{\log \frac{1}{1-r}}=\tau>1 .
$$

If $f$ and $g$ share $a_{j}(j=1,2, \ldots, 5)$ IM in $\Delta\left(\theta_{0}, \delta\right)$, then $f(z) \equiv g(z)$.

REMARK 1.2. Let $f$ be a meromorphic function in the unit disc. If for arbitrary small $\varepsilon>0$, we have

$$
\limsup _{r \rightarrow 1^{-}} \frac{\log n\left(r, \Delta\left(\theta_{0}, \varepsilon\right), f(z)=a\right)}{\log \frac{1}{1-r}}:=\tau
$$

for all but at most two $a \in \widehat{\mathrm{C}}$, then $e^{i \theta_{0}}$ is called a Borel point of order $\tau$ of $f(z)$. In [14], G. Valiron proved that every meromorphic function of finite order $\rho$ in the unit disc must have at least one Borel point of order $\rho+1$.

It is a natural question to ask whether we can relax the conditions of Theorem 1.7 on the sharing of values in $\Delta\left(\theta_{0}, \delta\right)$ ?

In this paper, we will answer the above question by studying the uniqueness problem for meromorphic functions in the unit disc $D$ sharing four distinct values and one set in an angular domain. We obtain the following results. 
THEOREM 1.8. Let $f, g$ be two meromorphic functions in $\mathrm{D}, a_{j} \in \widehat{\mathrm{C}}(j=$ $1,2,3,4)$ be four distinct values, and $\Delta\left(\theta_{0}, \delta\right)(0<\delta<\pi)$ be an angular domain satisfying (2). We assume that $f$ and $g$ share four distinct values $a_{j}$ $(j=1,2,3,4) I M$ in $\Delta\left(\theta_{0}, \delta\right)$ and $\bar{E}_{\Delta\left(\theta_{0}, \delta\right)}(S, f) \subset \bar{E}_{\Delta\left(\theta_{0}, \delta\right)}(S, g)$, where $S=\left\{b_{1}, \ldots, b_{m}\right\}, m \geq 1$ and $b_{1}, \ldots, b_{m} \in \widehat{C} \backslash\left\{a_{1}, a_{2}, a_{3}, a_{4}\right\}$. Then $f$ and $g$ share all values $C M$; thus it follows that either $f \equiv g$ or $f$ is a Möbius transformation of $g$. Furthermore, if the number of the values in $S$ is odd then $f \equiv g$.

REMARK 1.3. The special case $m=1$ of Theorem 1.8 immediately yields Theorem 1.7. In fact, when $m=1$, set $S=\left\{a_{5}\right\}$. If $f, g$ share $a_{5} I M$, which implies $\bar{E}_{\Delta\left(\theta_{0}, \delta\right)}(S, f) \subset \bar{E}_{\Delta\left(\theta_{0}, \delta\right)}(S, g)$, then by Theorem 1.8 , we can get $f \equiv g$.

\section{Some Lemmas}

To prove our results, we need the following lemmas.

LEMMA 2.1. Suppose that $f$ is an admissible meromorphic function in D. Let $P(f)=a_{0} f^{p}+a_{1} f^{p-1}+\cdots+a_{p}\left(a_{0} \neq 0\right)$ be a polynomial of $f$ with degree $p$, where the coefficients $a_{j}(j=0,1, \ldots, p)$ are constants, and let $b_{j}$ $(j=1,2, \ldots, q)$ be $q(q \geq p+1)$ distinct finite complex numbers. Then

$$
m\left(r, \frac{P(f) \cdot f^{\prime}}{\left(f-b_{1}\right)\left(f-b_{2}\right) \ldots\left(f-b_{q}\right)}\right)=S(r, f) .
$$

Proof. We can get this lemma by using the same argument as in Lemma 4.3 in [18].

LEMMA 2.2. Let $f$ and $g$ be two distinct admissible meromorphic functions in $\mathrm{D}$ and share four distinct values $a_{j}(j=1,2,3,4)$ IM in D. Let

$$
\varphi=\frac{f^{\prime} g^{\prime}(f-g)^{2}}{\left(f-a_{1}\right)\left(f-a_{2}\right)\left(f-a_{3}\right)\left(f-a_{4}\right)\left(g-a_{1}\right)\left(g-a_{2}\right)\left(g-a_{3}\right)\left(g-a_{4}\right)} \text {. }
$$

Then $T(r, \varphi)=S(r, f)+S(r, g)$ and

(i) $T(r, f) \leq T(r, g)+S(r, f), T(r, g) \leq T(r, f)+S(r, g)$;

(ii) $\sum_{j=1}^{4} \bar{N}\left(r, \frac{1}{f-a_{j}}\right)=2 T(r, f)+S(r, f)$, where $S(r):=S(r, f)=$ $S(r, g)$;

(iii) $\bar{N}\left(r, \frac{1}{f-b}\right)=T(r, f)+S(r, f), \bar{N}\left(r, \frac{1}{g-b}\right)=T(r, g)+S(r, g)$; where $b \neq a_{j}(j=1,2,3,4)$, and $\bar{N}\left(r, \frac{1}{f-b}\right)=\bar{N}(r, f)$ when $b=\infty$. 
Proof. We can get the conclusions of Lemma 2.2 by using the same argument as in Theorem 4.4 in [18].

Lemma 2.3. Suppose that $f$ and $g$ are distinct admissible meromorphic functions in D. If $f$ and $g$ share four distinct values $a_{j} \in \widehat{C}(j=1,2,3,4)$ $C M$ in $\mathrm{D}$, then $f$ is a Möbius transformation of $g$, two of the shared values, say $a_{1}$ and $a_{2}$, are Picard values in $\mathrm{D}$, and the cross ratio $\left(a_{1}, a_{2}, a_{3}, a_{4}\right)=-1$.

Proof. Proceeding as in the proof of Lemma 2.8 in [9], we can get the conclusions of Lemma 2.3 easily.

Lemma 2.4. Let $f, g$ be two admissible meromorphic functions in $\mathrm{D}, a_{j} \in$ $\widehat{\mathrm{C}}(j=1,2,3,4)$ be four distinct values, and $S=\left\{b_{1}, \ldots, b_{m}\right\}, m \geq 1$ and $b_{1}, \ldots, b_{m} \in \widehat{\mathrm{C}} \backslash\left\{a_{1}, a_{2}, a_{3}, a_{4}\right\}$. We assume that $f$ and $g$ share four distinct values $a_{j}(j=1,2,3,4) I M$ in $\mathrm{D}$ and $\bar{E}_{\mathrm{D}}(S, f) \subset \bar{E}_{\mathrm{D}}(S, g)$. Then $f$ and $g$ share all values $C M$ in $\mathrm{D}$, thus it follows that either $f \equiv g$ or $f$ is a Möbius transformation of $g$. Furthermore, if the number of the values in $S$ is odd, then $f \equiv g$.

Proof. Suppose that $f \not \equiv g$ and none of the $a_{j}(j=1,2,3,4)$ is $\infty$. Let $\varphi$ be the function expressed in Lemma 2.2. Then $\varphi \not \equiv 0$. By Lemma 2.2 (iii), we have

$$
m\left(r, \frac{1}{f-b_{j}}\right)=S(r, f), \quad m\left(r, \frac{1}{g-b_{j}}\right)=S(r, g),
$$

for any $b_{j} \in S(j=1,2, \ldots, m)$.

Set

$$
\varphi_{1}:=\frac{\left(g-b_{1}\right) \ldots\left(g-b_{m}\right)}{\left(f-b_{1}\right) \ldots\left(f-b_{m}\right)} \cdot\left(\frac{g^{\prime}(f-g)}{\left(g-a_{1}\right) \ldots\left(g-a_{4}\right)}\right)^{m}
$$

and

$$
\varphi_{2}:=\frac{\left(f-b_{1}\right) \ldots\left(f-b_{m}\right)}{\left(g-b_{1}\right) \ldots\left(g-b_{m}\right)} \cdot\left(\frac{f^{\prime}(f-g)}{\left(f-a_{1}\right) \ldots\left(f-a_{4}\right)}\right)^{m} .
$$

By Lemma 2.1 and (4), we can get that

$$
m\left(r, \frac{1}{f-b_{j}} \cdot \frac{g^{\prime}(f-g)\left(g-b_{j}\right)}{\left(g-a_{1}\right) \ldots\left(g-a_{4}\right)}\right)=S(r)
$$

and

$$
m\left(r, \frac{1}{g-b_{j}} \cdot \frac{f^{\prime}(f-g)\left(f-b_{j}\right)}{\left(f-a_{1}\right) \ldots\left(f-a_{4}\right)}\right)=S(r) .
$$

From the definitions of $\varphi_{1}$ and $\varphi_{2}$, we have $m\left(r, \varphi_{j}\right)=S(r), j=1,2$. By Lemma 2.2(iii), we see that "almost all" of poles and $b_{j}$-points of $f$ and $g$ in 
the unit disc D are simple. Since $f, g$ share the four distinct values $a_{j}, j=$ $1,2,3,4$ in the unit disc D and $\bar{E}_{\mathrm{D}}(S, f) \subset \bar{E}_{\mathrm{D}}(S, g)$, we can easily get that $N\left(r, \varphi_{1}\right)=S(r)$. Therefore, we have

$$
T\left(r, \varphi_{1}\right)=S(r)
$$

Since $\varphi_{1} \varphi_{2} \equiv \varphi^{m}$, we can have

$$
T\left(r, \varphi_{2}\right)=S(r)
$$

Let $L_{\mathrm{D}}^{p q}\left(a_{j}\right)$ be the set of those $a_{j}$-points of $f$ and $g$ in the unit disc D such that the multiplicities of $f$ and $g$ at these points are $p$ and $q$, respectively. For any $z_{0} \in L_{\mathrm{D}}^{p q}\left(a_{1}\right)$, by simple computation, we have

$$
\varphi_{1}\left(z_{0}\right)=\left(q \cdot \frac{f^{\prime}\left(z_{0}\right)-g^{\prime}\left(z_{0}\right)}{\left(a_{1}-a_{2}\right)\left(a_{1}-a_{3}\right)\left(a_{1}-a_{4}\right)}\right)^{m}
$$

and

$$
\varphi_{2}\left(z_{0}\right)=\left(p \cdot \frac{f^{\prime}\left(z_{0}\right)-g^{\prime}\left(z_{0}\right)}{\left(a_{1}-a_{2}\right)\left(a_{1}-a_{3}\right)\left(a_{1}-a_{4}\right)}\right)^{m} .
$$

Hence

$$
\frac{1}{q^{m}} \varphi_{1}\left(z_{0}\right)-\frac{1}{p^{m}} \varphi_{2}\left(z_{0}\right)=0 .
$$

Similarly, we can see that (7) holds for any $z_{0} \in L_{\mathrm{D}}^{p q}\left(a_{j}\right), j=2,3,4$.

Now we discuss two cases as follows.

CASE 1. Suppose that $\varphi^{p q}:=\frac{1}{q^{m}} \varphi_{1}-\frac{1}{p^{m}} \varphi_{2} \not \equiv 0$, for all positive integers $p, q$.

For the sake of convenience, we denote by $N^{p q}\left(r, \frac{1}{f-a_{j}}\right)$ the counting function of $f$ in the unit disc D with respect to the set $L_{\mathrm{D}}^{p q}\left(a_{j}\right)$, denote by $N^{p q}\left(r, \frac{1}{f-a_{j}}\right)$ the corresponding reduced counting function. Thus, we have

$$
N\left(r, \frac{1}{f-a_{j}}\right)=\sum_{p, q=1}^{\infty} N^{p q}\left(r, \frac{1}{f-a_{j}}\right)
$$

and

$$
N\left(r, \frac{1}{f-a_{j}}\right)=\sum_{p, q=1}^{\infty} N^{p q}\left(r, \frac{1}{f-a_{j}}\right) .
$$

From the above two equations, (4), (5) and (6), we can see that $T\left(r, \varphi^{p q}\right)=$ $S(r, f)+S(r, g)$. And by (7) each zero of $f-a_{j}$ is a zero of $\varphi^{p q}$, so with the 
help of $\varphi^{p q} \not \equiv 0$, we can get

$$
\begin{aligned}
\bar{N}^{p q}\left(r, \frac{1}{f-a_{j}}\right) & \leq \bar{N}^{p q}\left(r, \frac{1}{\varphi^{p q}}\right) \leq T\left(r, \frac{1}{\varphi^{p q}}\right) \leq T\left(r, \varphi^{p q}\right)+O(1) \\
& =S(r, f)+S(r, g):=S(r),
\end{aligned}
$$

for some $p, q$. By Lemma 2.2 (ii), we have $T(r, f)+S(r, f)=T(r, g)+$ $S(r, g)$. Thus, from the definition of $S(r)$, we can get $T(r, f)=T(r, g)+S(r)$. Therefore, we have

$$
\begin{aligned}
\bar{N}\left(r, \frac{1}{f-a_{j}}\right)= & \sum_{\max (p, q) \geq 5} \bar{N}^{p q}\left(r, \frac{1}{f-a_{j}}\right)+S(r, f) \\
\leq & \frac{1}{5}\left(\sum_{\max (p, q) \geq 5} N^{p q}\left(r, \frac{1}{f-a_{j}}\right)\right. \\
& \left.+\sum_{\max (p, q) \geq 5} N^{p q}\left(r, \frac{1}{g-a_{j}}\right)\right)+S(r, f) \\
\leq & \frac{1}{5}\left(N\left(r, \frac{1}{f-a_{j}}\right)+N\left(r, \frac{1}{g-a_{j}}\right)\right)+S(r, f) \\
\leq & \frac{2}{5} T(r, f)+S(r),
\end{aligned}
$$

for $j=1,2,3,4$.

By the above inequality and Lemma 2.2 (ii), we can get

$$
2 T(r, f) \leq \frac{8}{5} T(r, f)+S(r) .
$$

Thus, we can get a contradiction.

CASE 2. Suppose that $\varphi^{p q}:=\frac{1}{q^{m}} \varphi_{1}-\frac{1}{p^{m}} \varphi_{2} \equiv 0$, for some positive integers $p, q$. From the definitions of $\varphi_{1}$ and $\varphi_{2}$, we have

$$
\left(\frac{p}{q}\right)^{m} \cdot \frac{\left(g-b_{1}\right)^{2} \ldots\left(g-b_{m}\right)^{2}}{\left(f-b_{1}\right)^{2} \ldots\left(f-b_{m}\right)^{2}} \equiv\left(\frac{f^{\prime}\left(g-a_{1}\right) \ldots\left(g-a_{4}\right)}{g^{\prime}\left(f-a_{1}\right) \ldots\left(f-a_{4}\right)}\right)^{m} .
$$

Next we take the two subcases in the following into consideration:

SubCaSe 2.1. Suppose that $p \neq q$. Without loss of generality, we may assume that $p<q$. For some two positive integers $p_{1}$ and $q_{1}$, if $z_{1} \in L_{\mathrm{D}}^{p_{1} q_{1}}\left(a_{j}\right)$ for some $j \in\{1,2,3,4\}$, then (9) implies that $\frac{p}{q}=\frac{p_{1}}{q_{1}}$. Hence $q_{1}>p_{1} \geq 1$, 
and $q_{1} \geq 2$ which means that any $a_{j}$-points $(j=1,2,3,4)$ of $g$ in the unit disc $\mathrm{D}$ are multiple. By Lemma 2.2, we can get

$$
\begin{aligned}
2 T(r, g)=\sum_{j=1}^{4} \bar{N}\left(r, \frac{1}{g-a_{j}}\right)+S(r, g) & \leq \frac{1}{2} \sum_{j=1}^{4} N\left(r, \frac{1}{g-a_{j}}\right)+S(r, g) \\
& \leq 2 T(r, g)+S(r, g),
\end{aligned}
$$

which leads to the following equations

$$
T(r, g)=N\left(r, \frac{1}{g-a_{j}}\right)+S(r),
$$

and

$$
N\left(r, \frac{1}{g-a_{j}}\right)=2 \bar{N}\left(r, \frac{1}{g-a_{j}}\right)+S(r) .
$$

From (10) and (11), we can see that "almost all" of $a_{j}$-points of $g$ have multiplicity 2, and "almost all" of $a_{j}$-points of $f$ are simple in the unit disc D. Without loss of generality, we may assume that $f$ and $g$ attain the values $a_{3}$ and $a_{4}$ in the unit disc D. Set

$$
\phi_{1}:=\frac{2 f^{\prime}\left(f-a_{4}\right)}{\left(f-a_{1}\right)\left(f-a_{2}\right)\left(f-a_{3}\right)}-\frac{g^{\prime}\left(g-a_{4}\right)}{\left(g-a_{1}\right)\left(g-a_{2}\right)\left(g-a_{3}\right)}
$$

and

$$
\phi_{2}:=\frac{2 f^{\prime}\left(f-a_{3}\right)}{\left(f-a_{1}\right)\left(f-a_{2}\right)\left(f-a_{4}\right)}-\frac{g^{\prime}\left(g-a_{3}\right)}{\left(g-a_{1}\right)\left(g-a_{2}\right)\left(g-a_{4}\right)} .
$$

Since $\phi_{i}(i=1,2)$ is analytic at the poles of $f$ and of $g$ and also at those common $a_{j}$-points of $f$ and $g$ which have multiplicity 1 with respect to $f$ and multiplicity 2 with respect to $g$, by Lemma 2.1, we have $T\left(r, \phi_{i}\right)=S(r, f), i=1,2$. If $\phi_{i} \not \equiv 0$, then $N\left(r, \frac{1}{f-a_{4}}\right) \leq N\left(r, \frac{1}{\phi_{1}}\right)=S(r, f)$, which contradicts to equation (10). Then $\phi_{1} \equiv 0$. Similarly, we have $\phi_{2} \equiv 0$. Therefore, from the definitions of $\phi_{1}$ and $\phi_{2}$, we have

$$
\left(\frac{f-a_{4}}{f-a_{3}}\right)^{2} \equiv\left(\frac{g-a_{4}}{g-a_{3}}\right)^{2} .
$$

Since $f \not \equiv g$, and from (12), we have

$$
\frac{f-a_{4}}{f-a_{3}} \equiv-\frac{g-a_{4}}{g-a_{3}}
$$


which implies that $f$ and $g$ share $a_{3}, a_{4} C M$ in the unit disc D. Since $f$ and $g$ assume the value $a_{3}$ there exist positive integers $p_{1}, q_{1}$ such that $L_{\mathrm{D}}^{p_{1} q_{1}}\left(a_{3}\right) \neq \emptyset$. From the considerations above we get $q_{1}>p_{1}$, contradicting the fact that $f$ and $g$ share $a_{3} C M$.

SubCaSe 2.2. Suppose that $p=q$.

In this subcase, (10) becomes

$$
\frac{\left(g-b_{1}\right)^{2} \ldots\left(g-b_{m}\right)^{2}}{\left(f-b_{1}\right)^{2} \ldots\left(f-b_{m}\right)^{2}} \equiv\left(\frac{f^{\prime}\left(g-a_{1}\right) \ldots\left(g-a_{4}\right)}{g^{\prime}\left(f-a_{1}\right) \ldots\left(f-a_{4}\right)}\right)^{m} .
$$

which implies that $f$ and $g$ share the four values $a_{j}(j=1,2,3,4) C M$ in the unit disc D. From the conditions of this lemma and applying Lemma 2.3, $g$ is a Möbius transformation of $f$. Furthermore, two of the four values, say $a_{1}, a_{2}$ are Picard exceptional values of $f$ and $g$ in the unit disc D. Set

$$
\chi_{1}:=\frac{f^{\prime}\left(f-a_{4}\right)}{\left(f-a_{1}\right)\left(f-a_{2}\right)\left(f-a_{3}\right)}-\frac{g^{\prime}\left(g-a_{4}\right)}{\left(g-a_{1}\right)\left(g-a_{2}\right)\left(g-a_{3}\right)}
$$

and

$$
\chi_{2}:=\frac{f^{\prime}\left(f-a_{3}\right)}{\left(f-a_{1}\right)\left(f-a_{2}\right)\left(f-a_{4}\right)}-\frac{g^{\prime}\left(g-a_{3}\right)}{\left(g-a_{1}\right)\left(g-a_{2}\right)\left(g-a_{4}\right)} .
$$

Using the analogous argument of Subcase 2.1 for $\chi_{1}, \chi_{2}$, we can get

$$
\frac{f-a_{3}}{f-a_{4}} \equiv-\frac{g-a_{3}}{g-a_{4}} .
$$

We define the Möbius transformations $T, M$ and $L$ by

$$
T(\omega):=\frac{w-a_{3}}{w-a_{4}}, \quad M(w):=-w \quad \text { and } \quad L:=T^{-1} \circ M \circ T .
$$

Then we have

$$
T \circ f=-T \circ g, \quad \text { hence } \quad g=L \circ f .
$$

Obviously $a_{3}$ and $a_{4}$ are the fixed points of $L$. Therefore, there exist no fixed points of $L$ in the set $S$. Let some $b \in S$ be given. Then in view of $b \neq a_{1}, a_{2}$ there exists a $z_{0} \in \mathrm{C}$ such that $b=f\left(z_{0}\right)$, and from $\bar{E}_{\mathrm{D}}(S, f) \subseteq \bar{E}_{\mathrm{D}}(S, g)$ we obtain

$$
L(b)=L\left(f\left(z_{0}\right)\right)=g\left(z_{0}\right) \in S .
$$

So $S$ is invariant under $L$. Furthermore, we have $L \circ L=I$ where $I$ denotes the identical transformation. Hence we can conclude that $S$ must contain an even number of values. 
Thus, we complete the proof of Lemma 2.4.

Lemma 2.5 ([9]). Set

$$
u=u(z)=\frac{z^{\frac{\pi}{\delta}}+2 z^{\frac{\pi}{2 \delta}}-1}{z^{\frac{\pi}{\delta}}-2 z^{\frac{\pi}{2 \delta}}-1} .
$$

Then $u(z)$ maps conformally $\{z:|\arg z|<\delta,|z|<1\}$ onto the unit disc $\{u:|u|<1\}$, where $0<\delta<\pi$.

\section{Proof of Theorem 1.8}

Without loss of generality, we may assume $\theta_{0}=0$. Set

$$
u=u(z)=\frac{z^{\frac{\pi}{\delta}}+2 z^{\frac{\pi}{2 \delta}}-1}{z^{\frac{\pi}{\delta}}-2 z^{\frac{\pi}{2 \delta}}-1} .
$$

Let $z=z(u)$ denote its inverse function. Then by Lemma 2.5 we know that $u$ maps conformally $\Delta(0, \delta)$ onto the unit disc $\{u:|u|<1\}$.

Using the same method as in the proof of Theorem 1.2 in [10], we can get two meromorphic functions $f\left(z(u)\right.$ and $g(z(u))$ in the unit disc $\mathrm{D}^{\prime}=\{u:|u|<1\}$, and $f(z(u))$ is admissible in the disc $\mathrm{D}^{\prime}=\{u:|u|<1\}$. For the convenience of the reader, we describe the proof of this lemma as follows.

Set $z_{0}=p e^{i \vartheta} \in \Delta(0, \delta)$, by (13) we get (14)

$$
\begin{aligned}
1-\left|u\left(z_{0}\right)\right|=1-\sqrt{\frac{A^{2}+B^{2}}{C^{2}+D^{2}}} & =\frac{C^{2}+D^{2}-A^{2}-B^{2}}{C^{2}+D^{2}+\sqrt{\left(A^{2}+B^{2}\right)\left(C^{2}+D^{2}\right)}} \\
& =\frac{8 p^{\frac{\pi}{2 \delta}}\left(1-p^{\frac{\pi}{\delta}}\right) \cos \frac{\pi \vartheta}{2 \delta}}{C^{2}+D^{2}+\sqrt{\left(A^{2}+B^{2}\right)\left(C^{2}+D^{2}\right)}},
\end{aligned}
$$

where

$$
\begin{array}{ll}
A=p^{\frac{\pi}{\delta}} \cos \frac{\pi \vartheta}{\delta}+2 p^{\frac{\pi}{2 \delta}} \cos \frac{\pi \vartheta}{2 \delta}-1, & B=p^{\frac{\pi}{\delta}} \sin \frac{\pi \vartheta}{\delta}+2 p^{\frac{\pi}{2 \delta}} \sin \frac{\pi \vartheta}{2 \delta}, \\
C=p^{\frac{\pi}{\delta}} \cos \frac{\pi \vartheta}{\delta}-2 p^{\frac{\pi}{2 \delta}} \cos \frac{\pi \vartheta}{2 \delta}-1, & D=p^{\frac{\pi}{\delta}} \sin \frac{\pi \vartheta}{\delta}-2 p^{\frac{\pi}{2 \delta}} \sin \frac{\pi \vartheta}{2 \delta} .
\end{array}
$$

Since

$$
C^{2}+D^{2}=p^{\frac{2 \pi}{\delta}}+2 p^{\frac{\pi}{\delta}}+1+4 p^{\frac{2 \pi}{\delta}}\left(1-p^{\frac{\pi}{\delta}}\right) \cos \frac{\pi \vartheta}{2 \delta}+2 p^{\frac{\pi}{\delta}}\left(1-\cos \frac{\pi \vartheta}{\delta}\right),
$$

we can get

$$
1 \leq C^{2}+D^{2} \leq C^{2}+D^{2}+\sqrt{\left(A^{2}+B^{2}\right)\left(C^{2}+D^{2}\right)} \leq 2\left(C^{2}+D^{2}\right) \leq 20 .
$$


Since $\lim _{p \rightarrow 1^{-}} \frac{1-p^{\frac{\pi}{\delta}}}{1-p}=\frac{\pi}{\delta}$, then there exists $b \in\left(\left(\frac{1}{2}\right)^{\frac{2 \delta}{\pi}}, 1\right)$ such that for all $p$ satisfying $b<p<1$, we have

$$
\frac{1}{2}<p^{\frac{\pi}{2 \delta}}<1, \quad \frac{\pi}{2 \delta}(1-p)<1-p^{\frac{\pi}{\delta}}<\frac{3 \pi}{2 \delta}(1-p) .
$$

Hence, from (14)-(16), we can get

$$
\min \left\{1-\left|u\left(p e^{i \vartheta}\right)\right|: b<p<r,|\vartheta|<\frac{\delta}{2}\right\}>\frac{\pi}{20 \delta}(1-r)
$$

for all $r \in(b, 1)$.

We will prove that $f(z(u))$ is admissible in the unit disc $\{u:|u|<1\}$ as follows.

From (2), we get that there exists a sequence $\left\{r_{n}\right\}$ of positive numbers such that $r_{n} \rightarrow 1$ for $n \rightarrow+\infty$ and such that for $\tau_{1}$ we have

$$
n\left(r_{n}, \Delta(0, \delta / 2), f(z)=a\right)>\left(\frac{1}{1-r_{n}}\right)^{\tau_{1}}
$$

for sufficiently large $n$ and $\tau>\lambda_{1}>1$.

Then from (18) and Theorem 1.3.2 in [7, pp. 16-17], we have

$$
\limsup _{t \rightarrow 1} \frac{T(t, f(z(u)))}{\log \frac{1}{1-t}} \geq \limsup _{t_{n}^{\prime} \rightarrow 1} \frac{T\left(t_{n}^{\prime}, f(z(u))\right)}{\log \frac{1}{1-t_{n}^{\prime}}} \geq \infty .
$$

Since $f(z(u))$ a meromorphic function in $\{u:|u|<1\}$, from (19), we get that $f(z(u))$ is admissible in the unit disc $\{u:|u|<1\}$.

From the assumption of Theorem 1.8, we can get that $f(z(u))$ and $g(z(u))$ share $a_{1}, a_{2}, a_{3}, a_{4} I M$ in $\mathrm{D}^{\prime}$ and $\bar{E}_{\mathrm{D}^{\prime}}(S, f(z(u)))=\bar{E}_{\mathrm{D}^{\prime}}(S, g(z(u)))$. Thus, from Lemma 2.4, we can get that $g(z(u))$ is admissible in $\mathrm{D}^{\prime}$.

Therefore, by Lemma 2.4, we can get the conclusions of Theorem 1.8.

Thus, we complete the proof of Theorem 1.8.

\section{REFERENCES}

1. Bank, S., A general theorem concerning the growth of solutions of first-order algebraic differential equations, Compositio Math. 25 (1972), 61-70.

2. Cao, T.-B., and Yi, H.-X., Analytic functions sharing three values DM in one angular domain, J. Korean Math. Soc. 45 (2008), 1523-1534.

3. Cao, T.-B., and Yi, H.-X., On the uniqueness of meromorphic functions that share four values in one angular domain, J. Math. Anal. Appl. 358 (2009), 81-97.

4. Fang, M.-L., Uniqueness of admissible meromorphic functions in the unit disc, Sci. China (A) 42 (1999), 367-381. 
5. Hayman, W. K., Meromorphic Functions, Oxford Univ. Press, London 1964.

6. Heittokangas, J., On complex differential equations in the unit disc, Ann. Acad. Sci. Fenn. Math. Diss. 122 (2000), 1-54.

7. Juneja, O. P., and Kapoor, G. P., Analytic Functions - Growth Aspects, Pitman Adv. Publ. Program, Pitman, Boston 1985.

8. Lin, W.-C., Mori, S., and Tohge, K., Uniqueness theorems in an angular domain, Tohoku Math. J. 58 (2006), 509-527.

9. Lin, W.-C., Mori, S., and Yi, H.-X., Uniqueness theorems of entire functions with shared-set in an angular domain, Acta Math. Sin. 24 (2008), 1925-1934.

10. Mao, Z.-Q., and Liu, H.-F., Meromorphic functions in the unit disc that share values in an angular domain, J. Math. Anal. Appl. 359 (2009), 444-450.

11. Nevanlinna, R., Le théorème de Picard-Borel et la théorie des fonctions méromorphes, Chelsea, New York 1974.

12. Reinders, M., A new characterization of Gundersen's example of two meromorphic functions sharing four values, Results Math. 24 (1993), 174-179.

13. Titzhoff, F., Slowly growing functions sharing values, Fiz. Mat. Fak. Moksl. Semin. Darb. 8 (2005), 143-164.

14. Valiron, G., Points de Picard et points de Borel des fonctions méromorphes dans un cercle, Bull. Sci. Math. (2) 56 (1932), 10-32.

15. Wu, Z.-J., and Sun, D.-C., A remark on uniqueness theorems in an angular domain, Proc. Japan Acad. (A) 84 (2008), 73-77.

16. Xu, H.-Y., and Cao, T.-B., Uniqueness of two analytic functions sharing four values in an angular domain, Ann. Polon. Math. 99 (2010), 55-65.

17. Xu, J.-F., and Yi, H.-X., On uniqueness of meromorphic functions with shared four values in some angular domains, Bull. Malays. Math. Sci. Soc. (2) 31 (2008), 57-65.

18. Yi, H.-X., Yang, C.-C., Uniqueness Theory of Meromorphic Functions, Science Press/Kluwer, Beijing/Dordrecht, 2003.

19. Yang, L., Value Distribution Theory, Springer/Science Press, Berlin/Beijing, 1993/1982.

20. Zhang, Q.-C., Meromorphic functions sharing values in an angular domain, J. Math. Anal. Appl. 349 (2009), 100-112.

21. Zheng, J.-H., On uniqueness of meromorphic functions with shared values in some angular domains, Canad J. Math. 47 (2004), 152-160.

22. Zheng, J.-H., On uniqueness of meromorphic functions with shared values in one angular domain, Complex Var. Theory Appl. 48 (2003), 777-785.

DEPARTMENT OF INFORMATICS AND ENGINEERING JINGDEZHEN CERAMIC INSTITUTE

JINGDEZHEN, JIANGXI 333403

CHINA

E-mail: xhyhhh@126.com

DEPARTMENT OF MATHEMATICS

NANCHANG UNIVERSITY

NANCHANG, JIANGXI 330031

CHINA

E-mail: tbcao@ncu.edu.cn or ctb97@163.com
INSTITUTE OF MATHEMATICS AND INFORMATICS JIANGXI NORMAL UNIVERSITY

NANCHANG, JIANGXI 330022

CHINA

E-mail: yicaifeng55@163.com 